\title{
Dispatch then Stop: Optimal Dissemination of Security Patches in Mobile Wireless Networks
}

\author{
M.H. R Khouzani,Saswati Sarkar, Eitan Altman
}

\begin{abstract}
The security threat posed by malware in mobile wireless networks can be countered through immunization using security patches. The distribution of patches however consumes bandwidth which is specially scarce in wireless networks, and must therefore be judiciously controlled in order to attain desired trade-offs between security risks and resource consumption. The desired tradeoffs can be attained by activating at any given time only fractions of dispatchers and selecting their packet transmission rates. We formulate the above trade-offs as optimal control problems that seek to minimize the aggregate network costs that depend on security risks and resource consumed by the countermeasures. We prove that the optimal control strategies have simple structures. When the resource consumption cost rate is concave, the control strategies are bang-bang with at most one jump from the maximum to the minimum value. When the resource consumption cost rate is convex, the above transition is strict but continuous.
\end{abstract}

\section{INTRODUCTION}

a) Motivation: Self-propagating malicious codes, referred to as worms, have represented a persistent threat against networks. Worms can eavesdrop, analyze the data traversing the network, access privileged information, hijack sessions, disrupt network functionalities such as routing, etc. In addition, a worm can kill a node, that is, it can render an infective node dysfunctional by inflicting irretrievable damage. The threat of malware is but more dire in mobile wireless networks in which the network resources are inherently more constrained, and the initial intrusion is easier, as the media is shared.

Worms spread through message transmission from infected to susceptible nodes. This spread of contagion through contact can be countered by immunization and healing. Specifically, the underlying vulnerability utilized by the worm, can be amended by installing security patches [1] that immunize the susceptible, and heal and immunize the infective nodes. However, the distribution of the patches burdens the limited resources in the network, and hence if not carefully controlled, can become a menace itself.

We consider non-replicative and replicative settings for dissemination of the security patches in a mobile wireless network. In the non-replicative model, a number of mobile (or stationary) nodes, referred to as dispatchers, are pre-loaded with the security patch, and deliver the patch to other nodes upon contact. The susceptible and infective receptors subsequently become immune to the contagion, and are referred

M. H. R Khouzani and Saswati Sarkar are at the Electrical and Systems Engineering Department of University of Pennsylvania. Their emails are $\{$ khouzani,swati\} @ seas.upenn.edu. Eitan Altman is at INRIA, Sophia Antipolis, France. His email is altmanesophia.inria.fr

The contributions of M. H. R. Khouzani and S. Sarkar have been supported by NSF grants 0621782, 0721308, 0914955, 0915203, 0915697. to as recovered. In the replicative model, the receptors, in addition, become dispatchers themselves - thus the dispatchers replicate. In each model, the tradeoff between resource consumption and security can be controlled by activating only a desired fraction of dispatchers and also regulating the rate at which they transmit packets.

b) Contributions: First, we model the dynamics of the spread of the worm in mobile wireless networks in presence of arbitrary dynamic dispatcher activation and transmission rate control policies, and quantify the costs associated with the corresponding security risks and resource consumptions (§II). This formulates the dynamic activation of the dispatchers and setting their communication rates as an optimal control problem that seeks to minimize the above overall cost.

Second, in both non-replicative and replicative models, we prove that optimal policies have simple structures: for a concave resource consumption cost rate, activate all dispatchers and choose the maximum possible transmission rate for them until a certain time; subsequently all dispatchers must be de-activated until the end of the network operation period $(\S \S$ III,IV). We have therefore shown that the optimal control is bang-bang with at most one jump that terminates at the minimum possible value. The optimal control has a similar structure for a strictly convex resource consumption cost rate, except that its transition from the maximum to minimum values is (strict but) continuous rather than abrupt.

c) Related Works: [1] and [2] consider both replicative and non-replicative dispatch in wired networks. The analytical tools and the results presented there do not however apply in our context since (i) the patching rate is assumed constant in [1], [2], whereas we consider dynamic patching policies and (ii) [1], [2] consider the final (maximum, resp.) number of the infective nodes as the performance metric whereas we investigate more general cost functions based on the level of infection as well as the overall bandwidth consumed by the dispatchers.

Very few research works have in fact tried to adopt the malware propagation models to investigate an optimal dynamic countermeasure response based on a quantified cost function in wired or wireless networks; [4]-[6] constitute some notable exceptions. [4] investigates a different counter-measure: that of reduction of reception gain of wireless nodes for slowing down the spread of malware in wireless networks. Our work differs from [5], [6] in that we consider (i) both replicative and non-replicative patching, (ii) more general network state evolution dynamics in that the counter-measure involves both immunization and healing, moreover the worm may cause mortality, and (iii) cost functions which are only assumed to be either concave or convex and therefore more general than 
quadratic functions in [5], [6]. Also unlike [5] we do not use any linearization of the system which can be very poor in the context of epidemic behaviour. Investigation of optimal solutions in our context thus require different analytical arguments.

\section{SySTEM MODEL}

\section{A. non-replicative dispatch}

A susceptible node is a mobile wireless device which is not contaminated by the worm, yet is vulnerable to infection. A node is infective if it is contaminated by the worm. An infective spreads the worm to a susceptible while transmitting data or control messages to it. The worm can kill an infective host, i.e., render it completely dysfunctional - such nodes are denoted dead. A functional node that is immune to the worm is referred to as recovered. A fraction $R_{0}$ of mobile nodes, referred to as dispatchers, are pre-loaded with security patches. The dispatchers are immune to infection themselves and are therefore always in the recovered state. The dispatchers can transmit the patches to the susceptible and infective nodes and immunize the susceptibles and possibly heal the infectives to the recovered state.

Let the total number of nodes in the network be $N$. Let the fraction of susceptible, infective, recovered and dead nodes at time $t$ be denoted by $S(t), I(t), R(t)$ and $D(t)$, respectively. Then, $S(t)+I(t)+R(t)+D(t)=1$. One can therefore represent the system using any three of the above states: we choose $(S, I, D)$. At the start of the recovery process, that is at time zero, some but not all nodes are infected: $0<I(0)=$ $I_{0}<1$, and WLoG only the dispatchers are in the recovered state: $R(0)=R_{0}, 0<R_{0}<1, I_{0}+R_{0}<1$, and WLoG no node is dead: $D(0)=0$. Thus, $S(0)=1-I_{0}-R_{0}$.

All functional nodes are assumed to roam in a vast 2-D region of area $A$ with an average velocity $v$. An infective transmits a message to a susceptible with a given probability whenever the two are in contact, that is, the infective detects the presence of the susceptible in its transmission range. Under mobility models such as the random waypoint or random direction model [7], Groenevelt et al. [8] have shown that the time between consecutive contacts of a specific pair of nodes is nearly exponentially distributed whose rate can be represented as $\hat{\beta} / A$, where $\hat{\beta}$ is a constant proportional to the communication range and average relative speed of the nodes. The worm at an infective node kills the host (by invoking specific codes) after an exponential random time with rate $\delta$, a parameter of the worm.

A dispatcher comes into contact with another node also after exponentially distributed random time with possibly different parameter $\tilde{\beta} / A$. Let the fraction of activated dispatchers at time $t$ be $\varepsilon(t)$, and each scans the media at rate $u(t)$ (i.e., $u(t)$ is the rate of transmission of scanning packets). Upon a contact between an activated dispatcher and another node, the security patch is transmitted from the dispatcher to the receiver node. If the receptor is a susceptible node, it installs the security patch, is subsequently immunized, and its state changes to recovered. If however the receptor is an infective, the patch may fail to heal it, or, the worm may prevent its installation. We capture the above possibility, by introducing a coefficient $0 \leq \pi \leq 1$.
Let $\vartheta(t):=u(t) \varepsilon(t)$, and let $\beta_{0}:=\hat{\beta} \times \lim _{N \rightarrow \infty} \frac{N}{A}, \beta_{1}:=$ $\tilde{\beta} \times \lim _{N \rightarrow \infty} \frac{N}{A}$, where $\lim _{N \rightarrow \infty} \frac{N}{A}$ is the node density. Now according to the results of [9], as $N$ grows, $S(t), I(t)$ and $D(t)$ converge to the solution of the following system of differential equations ${ }^{1}$ :

$$
\begin{aligned}
\dot{S}(t) & =-\beta_{0} I(t) S(t)-\beta_{1} \vartheta(t) R_{0} S(t) \\
\dot{I}(t) & =\beta_{0} I(t) S(t)-\pi \beta_{1} \vartheta(t) R_{0} I(t)-\delta I(t) \\
\dot{D}(t) & =\delta I(t)
\end{aligned}
$$

with initial constraints:

$$
I(0)=I_{0}, \quad S(0)=1-I_{0}-R_{0}, \quad D(0)=0
$$

and also satisfy the following constraints at all $t$ :

$$
0 \leq S(t), I(t), D(t), \quad S(t)+I(t)+D(t) \leq 1 .
$$

The network may suffer over time from the infected hosts, used by the worm to (i) eavesdrop and analyze and/or (ii) alter or destroy the traffic that is generated or relayed by the infected hosts. An attacker also inflicts cost by killing nodes. At each time $t$, the network incurs a cost at the rate of $f(I(t))$ due to the presence of the infectives, and $g(D(t))$ owing to the loss of nodes through mortality, where $f($.$) is a non-decreasing,$ twice-differentiable, convex function of $I$ such that $f(0)=0$ and $f(I)>0$ for $I>0, g($.$) is a non-decreasing differentiable$ function of $D$ such that $g(0)=0$.

Recall that there are $N R_{0}$ dispatchers in all, $\varepsilon(t)$ fraction of them are activated at time $t$, and these scan the media at rate $u(t)$. Thus the total rate of bandwidth consumed in scanning the media at time $t$ is directly proportional to $\varepsilon(t) u(t) R_{0}=$ $\vartheta(t) R_{0}$. The network incurs a cost at rate $h\left(R_{0} \vartheta(t)\right)$ due to the above bandwidth consumption, where $h(x)$ is a twicedifferentiable and increasing function in $x$ such that $h(0)=$ 0 and $h(x)>0$ when $x>0$. Note that the assumptions on $f(),. g(),. h($.$) are mild and natural and a large class of$ functions satisfy them.

The aggregate network cost therefore is: ${ }^{2}$

$$
J=\int_{0}^{T} f(I(t))+g(D(t))+h\left(R_{0} \vartheta(t)\right) d t .
$$

The system seeks to minimize the aggregate cost $J(\vartheta)$ by appropriately regulating the immunization rate function $\vartheta(t)$ subject to: $0 \leq \vartheta(t) \leq \vartheta_{\max }$ for all $t \in[0, T]$. The bounds on $\vartheta(t)$ arise since $0 \leq \varepsilon(t) \leq 1$ and $0 \leq u(t) \leq u_{\max }$ due to physical constraints of the dispatcher devices. With appropriate scaling by choice of $\beta_{1}$, we can assume $\vartheta_{\max }=1$. Thus,

$$
0 \leq \vartheta(t) \leq 1 \text { for all } t \in[0, T] .
$$

Definition 1: An immunization rate function $\underline{\vartheta}($.$) is called$ an admissible control if (i) $\underline{\vartheta}($.$) satisfies (5), and (ii) \underline{\vartheta}($. is piecewise continuous such that the left and right hand limits exist at the points of discontinuity. A pair of state and control functions $((S(),. I(),. D()),. \underline{\vartheta}()$.$) is called an$

\footnotetext{
${ }^{1}$ Throughout the paper, variables with dot marks (e.g., $\left.\dot{S}(t)\right)$ represent their time derivatives (e.g., time derivative of $S(t)$ ) and the prime signs (e.g., $f^{\prime}(I)$ ) designate their derivatives with respect to their argument (e.g., $I$ ).

${ }^{2}$ The cost function can also have terms depending on the final concentration of the infective and the dead nodes.
} 
admissible pair if (i) $\underline{\vartheta}($.$) is an admissible control and (ii) the$ pair satisfies (1), (2).

lem. 1: Any admissible pair of state and control functions $((S(t), I(t), D(t)), \underline{\vartheta}(t))$, satisfies the state constraints in (3) in $[0, T]$ interval. Moreover, all constraints except $D(t) \geq 0$ are satisfied in the strict form in $[0, T]$.

Outline of the proof: First, let $\delta>0$. The initial conditions in (2) ensure that all constraints in (3) are strictly met at $t=0$. except that $D(0)=0$. From (2) and the continuity of the state functions, $S(t), I(t)>0$, in an interval of positive length starting from $t=0$. Suppose this interval ends at $t_{1}<T$. But then, either $S\left(t_{1}\right)=0$ or $I\left(t_{1}\right)=0$, since $\dot{S}+\dot{I} \leq 0$ over this interval. Thus, from (1a), $\dot{S}(t) \geq-\left(\beta_{0}+\beta_{1}\right) S(t)$, and hence, $S(t) \geq S(0) e^{-\left(\beta_{0}+\beta_{1}\right) t}$, and similarly, $I(t) \geq$ $I(0) e^{-\left(\beta_{1}+\delta\right) t}$, in $\left(0, t_{1}\right)$. However, the continuity of $S, I$ imply that $S\left(t_{1}\right), I\left(t_{1}\right)>0$. Thus, $t_{1} \nless T$, and hence $S, I$ are positive throughout. From (1), $\frac{d}{d t}(S(t)+I(t)+D(t))<0$ at all $t \in(0, T]$ since $S(t), I(t)>0$. Also, $S(0)+I(0)+D(0)<$ 1. Thus, $S(t)+I(t)+D(t)<1$ at all $t \in(0, T]$. Finally, since $\delta>0$ and $I(t)>0$ at all $t, D(t)>0$ at all $t>0$, from (1c). The lemma follows. When $\delta=0$, the only difference is that $D(t)=0$ for all $t \in[0, T]$.

Since the state constraints in(3) are never active, we can pose an optimal control problem without any state constraints, which is, minimization of the cost in (4) through appropriate choice of admissible pair $((S, I, D), \underline{\vartheta})$.
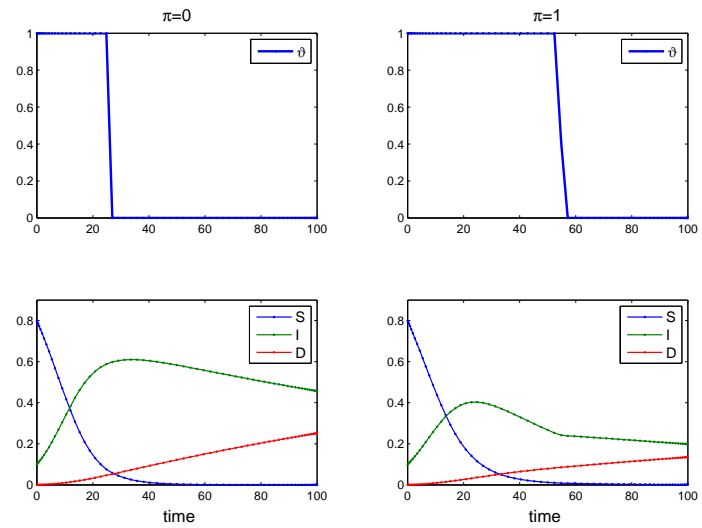

Fig. 1: The top figure represents the optimal control and the bottom figure the corresponding system states as functions of time for nonreplicative dispatch where $h($.$) is concave. Here, f(I)=10 I^{2}$, $g(D)=20 D^{2}, T=100, \beta_{0}=\beta_{1}=0.2, \delta=0.005$, $I_{0}=R_{0}=0.1, h(\vartheta)=10 R_{0} \vartheta$. Also, Left: $\pi=0$, Right: $\pi=1$.

\section{B. replicative dispatch}

The dynamics of state evolution in replicative dispatch differs from the non-replicative case in only that once a node receives a security patch, it can retransmit it upon contact with other nodes. Thus, all recovered nodes become dispatchers in the replicative model, and hence the fraction of dispatchers grows to $R(t)$ at time $t$ starting from the initial value of $R_{0}$, whereas the fraction of dispatchers continue to be $R_{0}$ at all times in the non-replicative model. Here, for convenience of analysis, we represent the system using

$$
\begin{aligned}
\dot{S}(t) & =-\beta_{0} I(t) S(t)-\beta_{1} \vartheta(t) R(t) S(t) \\
\dot{I}(t) & =\beta_{0} I(t) S(t)-\pi \beta_{1} \vartheta(t) R(t) I(t)-\delta I(t) \\
\dot{R}(t) & =\beta_{1} \vartheta(t) R(t) S(t)+\pi \beta_{1} \vartheta(t) R(t) I(t)
\end{aligned}
$$

with initial constraints:

$$
I(0)=I_{0}, \quad R(0)=R_{0}, \quad S(0)=1-I_{0}-R_{0},
$$

and as before $0<I_{0}, R_{0}, I_{0}+R_{0}<1$. Also,

$$
0 \leq S(t), I(t), R(t), \quad S(t)+I(t)+R(t) \leq 1 .
$$

Note that (6) differs from (1) in only that the equations for $\dot{S}(t)$ and $\dot{I}(t)$ have $R(t)$ instead of $R_{0}$.

The resource consumption cost incurred at time $t$ due to the bandwidth consumed in media scanning by the dispatchers is $h(R(t) \vartheta(t))$ (instead of $h\left(R_{0} \vartheta(t)\right)$ in the non-replicative case). Thus, the aggregate network cost is:

$$
J(\vartheta)=\int_{0}^{T}[f(I(t))+g(D(t))+h(R(t) \vartheta(t))] d t,
$$

where $D(t)=1-(S(t)+I(t)+R(t))$. Here, $f(),. g(),. h($. satisfy the same assumptions as before.

lem. 1 can be readily extended to the replicative case. Thus, the state constraints (8) are ignored henceforth and the optimal control problem can be posed similar to last paragraph of $\S I I-A$.

Remarks: Epidemic models (1), (6) demonstrate that in both non-replicative and replicative models, the state dynamics are non-linear differential equations and the state function $I(t)$ may be not monotonic.

Epidemic models similar to (1), (6) have been validated through experiments as well as network simulations to provide an acceptable representation of the spread of malware in mobile wireless networks (see e.g. [10], [11]).

Note that since $R(t) \geq R_{0}$ at all $t$, we can always (dynamically) choose the value of $\vartheta_{\text {rep }}$ in replicative setting so that $R \vartheta_{\text {rep }}$ is equal to $R_{0} \vartheta_{\text {non-rep }}$ and hence the aggregate cost under replicative dispatch is no higher than that under non-replicative dispatch. However, comparably, replicative dispatch is more vulnerable to contamination of the patches themselves as the number of dispatchers may grow exponentially. Note that since only the initial dispatchers transmit the patches in non-replicative setting, the system can counter this threat relatively easily by securing only these dispatchers.

In order to obtain fundamental bounds on the efficacy of the defense, we assume that the system computes the optimal immunization rate assuming full knowledge of the attack parameters, e.g., the killing rate $\delta$, the spread rates $\beta_{0}, \beta_{1}$ and healing efficacy of the patch $\pi$. We also assume that the values of these parameters do not change with time.

\section{Optimal Non-Replicative Dispatch}

We consider the optimal control problem posed by system dynamics of (1) and cost functional (4). First note that classical control techniques do not provide the optimal immunization rate in closed form since the state dynamics (1) is non-linear, 

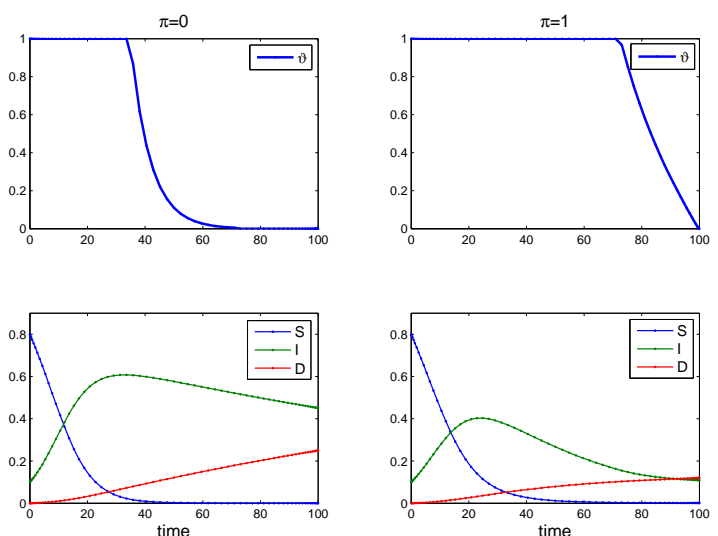

Fig. 2: The optimal control and the the corresponding system states as functions of time for non-replicative dispatch with strictly convex $h($.$) . The parameters are the same as in Fig. 1, except that h(\vartheta)=$ $10\left(R_{0} \vartheta\right)^{2}$. Also, Left: $\pi=0$, Right: $\pi=1$.

and the aggregate cost function (4) is not necessarily linear or quadratic.

Let $((S, I, D), \vartheta)$ be an optimal solution. Consider the Hamiltonian $H$, and co-state or adjoint functions $\lambda_{1}(t)$ to $\lambda_{3}(t)$ defined as follows:

$$
\begin{aligned}
& H=f(I)+g(D)+h\left(R_{0} \vartheta\right)+\left(\lambda_{2}-\lambda_{1}\right) \beta_{0} I S \\
& -\beta_{1} R_{0} \vartheta \lambda_{1} S-\pi \beta_{1} R_{0} \vartheta \lambda_{2} I+\left(\lambda_{3}-\lambda_{2}\right) \delta I . \\
& \dot{\lambda}_{1}=-\frac{\partial H}{\partial S}=-\left(\lambda_{2}-\lambda_{1}\right) \beta_{0} I+\beta_{1} R_{0} \vartheta \lambda_{1} \\
& \dot{\lambda}_{2}=-\frac{\partial H}{\partial I}=-f^{\prime}(I)-\left(\lambda_{2}-\lambda_{1}\right) \beta_{0} S \\
& +\pi \beta_{1} R_{0} \vartheta \lambda_{2}-\left(\lambda_{3}-\lambda_{2}\right) \delta \\
& \dot{\lambda}_{3}=-\frac{\partial H}{\partial D}=-g^{\prime}(D) .
\end{aligned}
$$

along with the transversality conditions:

$$
\lambda_{1}(T)=0, \quad \lambda_{2}(T)=0, \quad \lambda_{3}(T)=0 .
$$

Then according to Pontryagin's Maximum Principle ( [12, P. 109, Theorem 3.14]), there exist continuous and piecewise continuously differentiable state and co-state functions $S, I, D, \lambda_{1}, \lambda_{2}, \lambda_{3}$, that (i) satisfy (2), (12), and (ii) at every $t \in[0 \ldots T]$ where $\vartheta$ is continuous, satisfy (1), (11). Also,

$$
\vartheta \in \arg \min _{0 \leq \underline{\vartheta} \leq 1} H(\vec{\lambda},(S, I, D), \underline{\vartheta}) .
$$

Relation (13) between the optimum control $\vartheta$ and the Hamiltonian (10) allows us to express $\vartheta$ as a function of the state $(S, I, D)$ and co-state $\left(\lambda_{1}, \lambda_{2}, \lambda_{3}\right)$ functions in (1) and (11), resulting in a system of differential equations involving only the state and co-state functions, and not the control function. Using the initial and final values on the state and co-state functions, (2) and (12) respectively, this system can be solved numerically to obtain the optimum state and co-state functions, which can now be used to compute (i) $\vartheta$ via (13), (10) and (ii) $J(\vartheta)$ via (4).

\section{A. Structure of optimal non-replicative dispatch}

We now show that the optimal immunization rate function $\vartheta($.$) follows simple structures:$

Theorem 1: An optimal immunization rate function $\vartheta($. has the following structure:

1) When $h($.$) is concave, \vartheta(t)=1$ for $0<t<t_{1}$ and $\vartheta(t)=0$ for $t_{1}<t<T$.

2) When $h($.$) is strictly convex, \exists t_{0}, t_{1}, 0 \leq t_{0} \leq t_{1} \leq T$ :

a) $\vartheta(t)=1$ on $0<t \leq t_{0}$;

b) $\vartheta(t)$ strictly and continually decreases on $\left(t_{0}, t_{1}\right)$;

c) $\vartheta(t)=0$ on $t_{1} \leq t \leq T$.

Figures 1, 2 illustrate the optimum controls for linear and strictly convex $h($.$) respectively.$

Proof: Let $\varphi:=\beta_{1} R_{0}\left(\lambda_{1} S+\pi \lambda_{2} I\right)$ which is a continuous function of time, and by (12), $\varphi(T)=0$. Also, as we prove in $\S$ IIII-B,

lem. 2: $\varphi(t)$ is a strictly decreasing function of $t$ for $t \in$ $[0, T)$.

Now we can rewrite the Hamiltonian in (10) as:

$H=f(I)+g(D)+\left(\lambda_{2}-\lambda_{1}\right) \beta_{0} I S+\left(\lambda_{3}-\lambda_{2}\right) \delta I+h\left(R_{0} \vartheta\right)-\varphi \vartheta$.

From (13), for each admissible control $\underline{\vartheta}$, and for all $t \in[0, T]$,

$$
\begin{gathered}
h\left(R_{0} \vartheta(t)\right)-\varphi(t) \vartheta(t) \leq h\left(R_{0} \underline{\vartheta}(t)\right)-\varphi(t) \underline{\vartheta}(t) \\
\Longrightarrow \vartheta(t) \in \arg \min _{x \in[0,1]} h\left(R_{0} x\right)-\varphi(t) x .
\end{gathered}
$$

Also, since $\underline{\vartheta}=0$ is an admissible control, using (15),

$$
h\left(R_{0} \vartheta\right)-\varphi \vartheta \leq h(0)=0 \text { at all } t .
$$

We now separately consider the cases that $h($.$) is concave$ and strictly convex.

1) $h($.$) concave: When h^{\prime \prime} \leq 0$, at each time $t, h\left(R_{0} x\right)-$ $\varphi(t) x$ is a concave function of $x$, and thus a minimum in (16) is either at $x=0$ or $x=1$. Then,

$$
\vartheta(t)= \begin{cases}0, & \varphi(t)<h\left(R_{0}\right) \\ 1, & \varphi(t)>h\left(R_{0}\right) .\end{cases}
$$

According to $\varphi(T)=0$ and the continuity of $\varphi$ and since $h\left(R_{0}\right)>0$, we have $\varphi(t)<h\left(R_{0}\right)$ over a subinterval that extends to $T$. If this sub-interval starts from $t=0$, the theorem follows from (18) with $t_{1}=0$. Else, from the continuity of $\varphi$, and the Intermediate Value Theorem, $\varphi(t)=h\left(R_{0}\right)$ for some $t \in[0, T)$. But, there can be at most one such $t$, say $t_{1}$, by lem. 2. lem. 2 also implies that $\varphi(t)>h\left(R_{0}\right)$ for $t \in\left[0, t_{1}\right)$, and $\varphi(t)<h\left(R_{0}\right)$ for $t \in\left(t_{1}, T\right]$. The theorem follows from (18).

2) $h($.$) strictly convex: : When h($.$) is strictly convex (i.e.,$ $\left.h^{\prime \prime}>0\right)$, (16) implies that, if $\left.\frac{\partial}{\partial x}\left(h\left(R_{0} x\right)-\varphi(t) x\right)\right|_{x=y}=0$ at a $y \in[0,1]$, then $\vartheta(t)=y$, else $\vartheta(t) \in\{0,1\}$. Then,

$$
\vartheta= \begin{cases}0, & \varphi \leq R_{0} h^{\prime}(0) \\ \frac{1}{R_{0}} h^{\prime-1}\left(\varphi / R_{0}\right), & R_{0} h^{\prime}(0)<\varphi \leq R_{0} h^{\prime}\left(R_{0}\right) \\ 1, & R_{0} h^{\prime}\left(R_{0}\right)<\varphi .\end{cases}
$$

Note that $\varphi(T)=0 \leq R_{0} h^{\prime}(0)$, since $R_{0}>0$ and $h^{\prime}(x) \geq 0$ for all $x$. Also, since $h($.$) is strictly convex, h^{\prime}($.$) is a strictly$ 
increasing function - hence, since $R_{0}>0, h^{\prime}(0)<h^{\prime}\left(R_{0}\right)$. Thus, following lem. 2 , there exist $t_{0}, t_{1}, 0 \leq t_{0} \leq t_{1} \leq T$, such that $\varphi>R_{0} h^{\prime}\left(R_{0}\right)$ on $0<t \leq t_{0}, R_{0} h^{\prime}(0)<\varphi \leq$ $R_{0} h^{\prime}\left(R_{0}\right)$ on $t_{0}<t<t_{1}$, and $\varphi \leq R_{0} h^{\prime}(0)$ on $t_{1} \leq t \leq T$. The theorem now follows from (19).

\section{B. Proof of lem. 2}

The state and co-state functions, and hence the $\varphi$ function, are differentiable at each $t \in[0, T)$ at which the $\vartheta$ function is continuous. Since $\vartheta$ is piecewise continuous and $\varphi$ is continuous, the lemma follows if we can show that $\dot{\varphi}<0$ at each such $t$. Since $\beta_{1}, R_{0}>0$, at each such $t \in[0, T)$,

$$
\begin{array}{r}
\quad \frac{\dot{\varphi}}{\beta_{1} R_{0}}=\frac{1}{\beta_{1} R_{0}} \frac{d}{d t} \varphi=\dot{\lambda}_{1} S+\lambda_{1} \dot{S}+\pi \dot{\lambda}_{2} I+\pi \lambda_{2} \dot{I} \\
=-\left(\lambda_{2}-\lambda_{1}\right) \beta_{0} I S-(1-\pi) \lambda_{1} \beta_{0} I S-\pi f^{\prime}(I) I-\pi \lambda_{3} \delta I
\end{array}
$$

The right hand side is negative at each $t \in[0, T)$ from lem. 1, since $0 \leq \pi \leq 1, \beta_{0}, \beta_{1}>0, \delta \geq 0, f^{\prime}(x) \geq 0$ at each $x$, and because:

lem. 3: For all $0 \leq t<T$, we have $\lambda_{3} \geq 0, \lambda_{1}>0$, and $\left(\lambda_{2}-\lambda_{1}\right)>0$.

Proof: First, $\lambda_{3}(T)=0$ and at any $t \in[0, T]$ at which $\vartheta$ is continuous, $\dot{\lambda}_{3}(t)=-g^{\prime}(D(t)) \leq 0$. Thus, since $\vartheta$ is piecewise continuous, $\lambda_{3}(t) \geq 0$ for all $0 \leq t \leq T$. We prove the other two inequalities using the following real analysis properties (which we state without proof due to lack of space).

Property 1: Let $\psi(t)$ be a continuous and piecewise differentiable function of $t$. Let $\psi\left(t_{1}\right)=L$ and $\psi(t)>L$ for all $t \in\left(t_{1} \ldots t_{0}\right]$. Then $^{3} \dot{\psi}\left(t_{1}^{+}\right) \geq 0$.

Property 2: For any convex and differentiable function, $v(x)$, which is 0 at $x=0, v^{\prime}(x) x-v(x) \geq 0$ for all $x \geq 0$.

The system is autonomous, i.e., the Hamiltonian and the constraints on the control (5) do not have an explicit dependency on the independent variable $t$. Thus, [13, P.236]

$$
H\left(S, I, D, \vartheta, \lambda_{1}, \lambda_{2}, \lambda_{3}\right) \equiv \text { constant. }
$$

Thus, from (12), $H=H(T)=f(I(T))+g(D(T))+$ $h\left(R_{0} \vartheta(T)\right)$. Also, $\dot{D}=\delta I \geq 0$, and $g($.$) is a non-decreasing$ function, thus $g(D(T)) \geq g(D(t))$ for all $t \in[0 \ldots T]$. Hence:

$$
H-g(D(t)) \geq f(I(T))+h\left(R_{0} \vartheta(T)\right)>0 .
$$

The positivity follows since (i) according to lem. $1, I(T)>0$ and hence $f(I(T))>0$ and (ii) $h\left(R_{0} \vartheta(T)\right) \geq 0$.

We proceed in the following two steps:

Step-1. $\left(\lambda_{2}(T)-\lambda_{1}(T)\right)=0$ and $\dot{\lambda}_{2}(T)=\left(\dot{\lambda}_{2}(T)-\right.$ $\left.\dot{\lambda}_{1}(T)\right)=-f^{\prime}(I(T))<0$. Also, $\lambda_{1}(T)=\dot{\lambda}_{1}(T)=0$ and $\ddot{\lambda}_{1}(T)=-\lambda_{2}(T) \beta_{0} I(T)>0$. Therefore, $\lambda_{1}(t)$ and $\left(\lambda_{2}(t)-\lambda_{1}(t)\right)$ are positive in an open interval of nonzero length ending at $T$.

Step-2. Proof by contradiction. Let $t^{*} \geq 0$ be the last time before $T$ at which (at least) one of the other two inequality constraints is active, i.e.,

$$
\begin{array}{rrr}
\text { for } t^{*}<t<T: & \lambda_{1}(t)>0, & \left(\lambda_{2}(t)-\lambda_{1}(t)\right)>0 \\
\text { and, } \lambda_{1}\left(t^{*}\right)=0 & \text { OR } & \lambda_{2}\left(t^{*}\right)-\lambda_{1}\left(t^{*}\right)=0
\end{array}
$$

\footnotetext{
${ }^{3}$ For a general function $\psi(x)$, the notations $\psi\left(x_{0}^{+}\right)$and $\psi\left(x_{0}^{-}\right)$are defined as $\lim _{x \downarrow x_{0}} \psi(x)$ and $\lim _{x \uparrow x_{0}} \psi(x)$, respectively.
}

First, let $\lambda_{2}\left(t^{*}\right)-\lambda_{1}\left(t^{*}\right)=0$. Now, from (11) and (14),

$$
\begin{array}{r}
\left(\dot{\lambda}_{2}\left(t^{*+}\right)-\dot{\lambda}_{1}\left(t^{*+}\right)\right) \\
=-f^{\prime}(I)+\pi \beta_{1} R_{0} \vartheta \lambda_{2}-\left(\lambda_{3}-\lambda_{2}\right) \delta-\beta_{1} R_{0} \vartheta \lambda_{1} \\
-\frac{H}{I}+\frac{f(I)}{I}+\frac{g(D)}{I}+\frac{1}{I}\left(h\left(R_{0} \vartheta\right)-\varphi \vartheta\right)+\left(\lambda_{3}-\lambda_{2}\right) \delta \\
=\frac{1}{I}\left[f(I)-f^{\prime}(I) I\right]-\frac{H-g(D)}{I} \\
-(1-\pi) \beta_{1} R_{0} \vartheta \lambda_{1}+\frac{1}{I}\left(h\left(R_{0} \vartheta\right)-\varphi \vartheta\right)
\end{array}
$$

From the supposition on $t^{*}$ and continuity of $\lambda_{1}(t), \lambda_{1}\left(t^{*+}\right) \geq$ 0 . Now, $f(I)-f^{\prime}(I) I \leq 0$ because of Property 2 , since $f(x)$ is convex, $f(0)=0$ and $I>0$ at all $t$ by lem. 1. Thus, from (5),(17),(21) and (22), we observe that $\left.\left[\frac{d}{d t}\left(\lambda_{2}-\lambda_{1}\right)\right]\right|_{t^{*+}}<0$. This contradicts Property 1 . Hence, $\left(\lambda_{2}\left(t^{*+}\right)-\lambda_{1}\left(t^{*+}\right)\right)>0$. Now let $\lambda_{1}\left(t^{*}\right)=0$. Then from (11), $\left.\dot{\lambda}_{1}\right|_{t^{*+}}=-\left(\lambda_{2}-\lambda_{1}\right) \beta_{0} I$. Since $\left(\lambda_{2}\left(t^{*+}\right)-\lambda_{1}\left(t^{*+}\right)\right)>0$, and from lem. $1, \dot{\lambda}_{1}\left(t^{*+}\right)<0$. This contradicts Property 1 , and hence negates the existence of $t^{*}$. The lemma follows.

\section{Optimal Replicative Dispatch}

We seek to find an admissible $\vartheta(t)$ to minimize the cost function in (9) for the state dynamics (6) and initial state values (7). We again apply Pontryagin's Maximum Principle. Define the Hamiltonian as:

$$
\begin{array}{r}
H=f(I)+g(D)+h(R \vartheta)+\left(\lambda_{2}-\lambda_{1}\right) \beta_{0} I S \\
-\left(\lambda_{1}-\lambda_{3}\right) \beta_{1} \vartheta R S-\left(\lambda_{2}-\lambda_{3}\right) \pi \beta_{1} \vartheta R I-\lambda_{2} \delta I .
\end{array}
$$

where $D=1-(S+I+R)$, and the co-state functions as:

$$
\begin{gathered}
\dot{\lambda}_{1}=-\frac{\partial H}{\partial S}=-\left(\lambda_{2}-\lambda_{1}\right) \beta_{0} I+\left(\lambda_{1}-\lambda_{3}\right) \beta_{1} \vartheta R+g^{\prime}(D) \\
\dot{\lambda}_{2}=-\frac{\partial H}{\partial I}=- \\
\quad f^{\prime}(I)-\left(\lambda_{2}-\lambda_{1}\right) \beta_{0} S+\left(\lambda_{2}-\lambda_{3}\right) \pi \beta_{1} \vartheta R \\
\quad+\lambda_{2} \delta+g^{\prime}(D) \\
\dot{\lambda}_{3}=-\frac{\partial H}{\partial R}=\left(\begin{array}{c}
\left.\lambda_{1}-\lambda_{3}\right) \beta_{1} \vartheta S+\left(\lambda_{2}-\lambda_{3}\right) \pi \beta_{1} \vartheta I \\
-\vartheta h^{\prime}(R \vartheta)+g^{\prime}(D)
\end{array}\right.
\end{gathered}
$$

and the transversality conditions as:

$$
\lambda_{1}(T)=\lambda_{2}(T)=\lambda_{3}(T)=0 .
$$

Then according to Pontryagin's Maximum Principle ( [12, P. 109, Theorem 3.14]), there exist continuous and piecewise continuously differentiable state and co-state functions $S, I, R, \lambda_{1}, \lambda_{2}, \lambda_{3}$, that (i) satisfy (7), (25), and (ii) at every $t \in[0 \ldots T]$ where $\vartheta$ is continuous, satisfy (6), (24). Also,

$$
\vartheta \in \arg \min _{0 \leq \underline{\vartheta} \leq 1} H(\vec{\lambda},(S, I, R), \underline{\vartheta}) \text {. }
$$

\section{A. Structure of optimal replicative dispatch}

In this section, we show that the optimum control has the same structure as under non-replicative dispatch. Specifically, Theorem 1 holds. Note that the transition from 1 to 0 in the bang-bang optimal control should invariably occur earlier for replicative dispatch ( for concave $h($.$) ) This is because$ 
in replicative dispatch the number of dispatchers increases exponentially fast. Thus, more infectives and susceptibles are healed and immunized respectively in shorter duration allowing for smaller initial period of maximum rate immunization. Also, the exponential growth in the number of dispatchers may result in a huge cost due to $\vartheta$, if it is not shut down to zero earlier.

In the rest of the subsection, we outline the proof of Theorem 1 for replicative dispatch.

Proof: Define $\varphi:=\left(\lambda_{1}-\lambda_{3}\right) \beta_{1} R S+\left(\lambda_{2}-\lambda_{3}\right) \pi \beta_{1} R I$, which is a continuous function of time, and from (25), $\varphi(T)=$ 0 . The Hamiltonian in (23) can be rewritten as follows:

$$
H=f(I)+g(D)+\left(\lambda_{2}-\lambda_{1}\right) \beta_{0} I S-\lambda_{2} \delta I+h(R \vartheta)-\varphi \vartheta .
$$

From (26), for each admissible control $\underline{\vartheta}$, and $\forall t \in[0, T]$, $h(R(t) \vartheta(t))-\varphi(t) \vartheta(t) \leq h(R(t) \underline{\vartheta}(t))-\underline{\varphi}(t) \underline{\vartheta}(t)$, thus

$$
\vartheta(t) \in \arg \min _{x \in[0,1]} h(R(t) x)-\varphi(t) x .
$$

Also, since $\underline{\vartheta}=0$ is an admissible control, $[h(R \vartheta)-\varphi \vartheta] \leq$ 0 at all $t$.

As in the non-replicative dispatch, we consider the cases that $h($.$) is concave and strictly convex separately. In both, we will$ use the expression for $\dot{\varphi}$ at each $t$ at which $\vartheta$ is continuous, which we next obtain:

$$
\begin{array}{r}
\dot{\varphi}=\left(\dot{\lambda}_{1}-\dot{\lambda}_{3}\right) \beta_{1} R S+\left(\dot{\lambda}_{2}-\dot{\lambda}_{3}\right) \pi \beta_{1} R I+\left(\lambda_{1}-\lambda_{3}\right) \beta_{1} \dot{R} S \\
+\left(\lambda_{2}-\lambda_{3}\right) \pi \beta_{1} \dot{R} I+\left(\lambda_{1}-\lambda_{3}\right) \beta_{1} R \dot{S}+\left(\lambda_{2}-\lambda_{3}\right) \pi \beta_{1} R \dot{I}
\end{array}
$$

replacing from (6), (24) and simplifiying yields:

$$
\begin{array}{r}
=-\beta_{0} \beta_{1}(1-\pi) R I S\left(\lambda_{1}-\lambda_{3}\right)-\beta_{0} \beta_{1} R I S\left(\lambda_{2}-\lambda_{1}\right) \\
-\pi \beta_{1} f^{\prime}(I) R I+\pi \beta_{1} R I \delta \lambda_{3}+\dot{R} h^{\prime}(R \vartheta) .
\end{array}
$$

We will also use the following key properties of the costate functions, whose proof is similar to that of lem. 3 , and is omitted for brevity.

lem. 4: For all $0 \leq t<T$, we have $\left(\lambda_{2}-\lambda_{1}\right)>0,\left(\lambda_{1}-\right.$ $\left.\lambda_{3}\right)>0$ and $\lambda_{3} \leq 0$.

1) $h($.$) concave: When h($.$) is concave (i.e., h^{\prime \prime} \leq 0$ ), a minima in (28) is either at $x=0$ or $x=1$ at each time $t$, and this minima is unique unless $h(R)-\varphi=0$. Then,

$$
\vartheta= \begin{cases}0, & \varphi-h(R)<0 \\ 1, & \varphi-h(R)>0\end{cases}
$$

For the case of $h^{\prime \prime}<0$, whenever $h(R)-\varphi=0, \vartheta \in\{0,1\}$. Let $\psi(t)=\varphi(t)-h(R(t))$. Because $\varphi(T)=0$ and from (30) and since $h(R(T))>0, \psi<0$ over a subinterval that extends to $T$. We next show that for all $t \in[0, T), \psi(t)$ strictly decreases with increase in $t$. The rest of the proof is identical to that for concave $h($.$) in \S$ III-A1 (with $\psi$ instead of $\varphi$ and 0 instead of $h\left(R_{0}\right)$ in the arguments).

Since $\vartheta$ is piecewise continuous and $\varphi, h, R$ are continuous, it suffices to show that $\dot{\psi}$ is negative at any $t \in[0, T)$ at which $\vartheta$ is continuous. Referring to (29), at any such $t$ :

$$
\begin{array}{r}
\dot{\psi}=\dot{\varphi}-h^{\prime}(R) \dot{R} \\
=-\beta_{0} \beta_{1}(1-\pi) R I S\left(\lambda_{1}-\lambda_{3}\right)-\beta_{0} \beta_{1} R I S\left(\lambda_{2}-\lambda_{1}\right) \\
-\pi \beta_{1} f^{\prime}(I) R I+\pi \beta_{1} R I \delta \lambda_{3}-\dot{R}\left(h^{\prime}(R)-h^{\prime}(R \vartheta)\right)
\end{array}
$$

We only need to show that the right hand side is negative at each $t \in[0, T)$. Note that $\dot{R}\left(h^{\prime}(R)-h^{\prime}(R \vartheta)\right) \equiv 0$. This follows readily for $h^{\prime \prime} \equiv 0$ as then $h^{\prime}(R)-h^{\prime}(R \vartheta) \equiv 0$ for any value of $\vartheta$. When $h^{\prime \prime}<0$, as we argued in (30) and after, $\vartheta \in\{0,1\}$; now for $\vartheta=1, h^{\prime}(R)-h^{\prime}(R \vartheta)=0$ and for $\vartheta=0, \dot{R}=0$. The negativity follows from generalization of 1 for the replicative case and lem. 4 .

2) $h($.$) strictly convex: When h($.$) is strictly convex (i.e.,$ $\left.h^{\prime \prime}>0\right),(28)$ implies that, if $\left.\frac{\partial}{\partial x}(h(R(t) x)-\varphi(t) x)\right|_{x=y}=0$ at a $y \in[0,1]$, then $\vartheta(t)=y$, else $\vartheta(t) \in\{0,1\}$. Thus,

$$
\vartheta= \begin{cases}0, & \frac{\varphi}{R} \leq h^{\prime}(0) \\ \frac{1}{R} h^{\prime-1}\left(\frac{\varphi}{R}\right), & h^{\prime}(0)<\frac{\varphi}{R} \leq h^{\prime}(R) \\ 1, & h^{\prime}(R)<\frac{\varphi}{R} .\end{cases}
$$

Note that $\varphi(T) / R(T)=0 \leq h^{\prime}(0)$, from the fact that $\varphi(T)=0$, lem. 4 and since $h^{\prime}(x) \geq 0$ for all $x$. The rest of the proof is identical to that for strictly convex $h($.$) in$ $\S$ III-A2 provided we can show that $\psi=(\varphi / R)$ is a strictly decreasing function of time. Since $\vartheta$ is piecewise continuous, and $\psi$ is continuous, it suffices to show that $\dot{\psi}$ is negative at any $t \in[0, T)$ at which $\vartheta$ is continuous. At at any such $t$,

$$
\dot{\psi}=\frac{\dot{\varphi}-\dot{R} \frac{\varphi}{R}}{R}=\frac{\{\text { negative term }\}+\dot{R}\left[h^{\prime}(R \vartheta)-\frac{\varphi}{R}\right]}{R}
$$

The last equality follows from (29), and the negative term is $-\beta_{0} \beta_{1}(1-\pi) R I S\left(\lambda_{1}-\lambda_{3}\right)-\beta_{0} \beta_{1} R I S\left(\lambda_{2}-\lambda_{1}\right)$

$-\pi \beta_{1} f^{\prime}(I) R I+\pi \beta_{1} R I \delta \lambda_{3}$. The negativity of this term is established by generalized lem. 1 for propagative case and lem. 4. Now, from (32), $\dot{R}\left[h^{\prime}(R \vartheta)-\frac{\varphi}{R}\right] \leq 0$. Hence, $\dot{\psi}<0$.

\section{REFERENCES}

[1] M. VojnoviĆ and A. Ganesh, "On the effectiveness of automatic patching," in 2005 ACM workshop on Rapid malcode. ACM New York, pp. $41-50$.

[2] S. Shakkottai and R. Srikant, "Peer to peer networks for defense against internet worms," in 2006 workshop on Interdisciplinary systems approach in performance evaluation and design of computer \& communications sytems. ACM New York.

[3] R. Dantu, J. Cangussu, and A. Yelimeli, "Dynamic control of worm propagation," in ITCC 2004, vol. 1, 2004.

[4] M. Khouzani, E. Altman, and S. Sarkar, "Optimal Quarantining of Wireless Malware Through Power Control," in Fourth Symposium on Information Theory and Applications. UCSD, 2009.

[5] M. Bloem, T. Alpcan, and T. Başar, "Optimal and robust epidemic response for multiple networks," Control Engineering Practice, vol. 17, no. 5, pp. 525-533, 2009

[6] X. Yan and Y. Zou, "Optimal Internet Worm Treatment Strategy Based on the Two-Factor Model," ETRI JOURNAL, vol. 30, no. 1, p. 81, 2008.

[7] C. Bettstetter, "Mobility modeling in wireless networks: categorization, smooth movement, and border effects," Mobile Computing and Communications Review, vol. 5, no. 3, pp. 55-66, 2001.

[8] R. Groenevelt, P. Nain, and G. Koole, "The message delay in mobile ad hoc networks," Performance Evaluation, vol. 62, no. 1-4, pp. 210-228, 2005.

[9] T. Kurtz, "Solutions of ordinary differential equations as limits of pure jump Markov processes," Journal of Applied Probability, pp. 49-58, 1970.

[10] P. De, Y. Liu, and S. Das, "An epidemic theoretic framework for evaluating broadcast protocols in wireless sensor networks," in 4th IEEE International Conference on Mobile Ad-hoc and Sensor Systems.

[11] S. Tanachaiwiwat and A. Helmy, "VACCINE: War of the worms in wired and wireless networks," in IEEE INFOCOM, 2006, pp. 05-859.

[12] D. Grass, J. Caulkins, G. Feichtinger, G. Tragler, and D. Behrens, Optimal control of nonlinear processes: with applications in drugs, corruption, and terror. Springer Verlag, 2008.

[13] D. Kirk, Optimal Control Theory: An Introduction. Prentice Hall, 1970. 